\title{
Quantification of eosinophilic granule protein deposition in biopsies of inflammatory skin diseases by automated image analysis of highly sensitive immunostaining *
}

\author{
Peter Kiehl ${ }^{* *}$, Katrin Falkenberg, \\ Markus Vogelbruch and Alexander Kapp \\ Department of Dermatology and Allergology, \\ Hannover Medical University, Germany
}

\section{Received 27 July 1999}

Accepted 1 November 1999

Eosinophilic granulocytes are major effector cells in inflammation. Extracellular deposition of toxic eosinophilic granule proteins (EGPs), but not the presence of intact eosinophils, is crucial for their functional effect in situ. As even recent morphometric approaches to quantify the involvement of eosinophils in inflammation have been only based on cell counting, we developed a new method for the cell-independent quantification of EGPs by image analysis of immunostaining. Highly sensitive, automated immunohistochemistry was done on paraffin sections of inflammatory skin diseases with 4 different primary antibodies against EGPs. Image analysis of immunostaining was performed by colour translation, linear combination and automated thresholding. Using strictly standardized protocols, the assay was proven to be specific and accurate concerning segmentation in 8916 fields of 520 sections, well reproducible in repeated measurements and reliable over 16 weeks observation time. The method may be valuable for the cell-independent segmentation of immunostaining in other applications as well.

Keywords: Eosinophilic granule proteins, inflammation, image analysis, immunohistochemistry, quantification

\footnotetext{
*Parts of this study were presented at the 6th Congress of the European Society for Analytical Cellular Pathology, April 7-11, 1999, Heidelberg, Germany.

${ }^{* *}$ Corresponding author: Dr Peter Kiehl, Head of the Dermatohistopathology and Electron Microscopy Unit, Department of Dermatology and Allergology, Hannover Medical University, Ricklinger Str. 5, D-30449 Hannover, Germany. Tel./Fax: +49 5119246 201; E-mail:PKiehl@csi.com.
}

\section{Introduction}

Eosinophilic granulocytes are major effector cells in many diseases, but especially in allergic inflammation $[8,24]$. Many recent studies substantially increased our knowledge of eosinophil activation and the regulation of eosinophilia by cytokines, chemokines and other inflammatory mediators like IL-5, GMCSF, C5a, RANTES, Eotaxin and 5-oxo-eicosanoids $[7,13]$. Accordingly, the important involvement of eosinophils, especially in T-helper-cell type 2 reactions (Th2), e.g., in atopic dermatitis and allergic bronchial asthma, is well established. The participation of eosinophils in allergic inflammation is complex, but release of reactive oxygen species and toxic eosinophilic granule proteins (EGPs) followed by local propagation of inflammation and tissue damage is most important. This extracellular tissue deposition of EGPs, expressing complete cellular activation and degranulation, but not only the presence of intact eosinophils is crucial for their functional effect in situ.

Due to easy accessibility, tissue deposition of EGPs has been mainly studied in skin biopsies. It has been shown that cytolysis is likely the usual mechanism of eosinophilic granule protein release [6]. In tissue sections, eosinophils are characterized and identified by the staining properties of their specific granules. Consequently, eosinophils are usually no longer identifiable in tissue after complete activation and degranulation, and conversely, visible eosinophils are not likely to be completely activated. Thus, eosinophil involvement in inflammation can not be judged by the number of intact eosinophils. As has been shown by immunofluorescence studies, at least in many inflammatory skin diseases, the deposition of granule proteins is vastly out of proportion to the number of identifiable cells [14]. 
In contrast to these findings, almost all morphometric approaches to quantify eosinophilia in tissue are based on cell counting, even very recent ones using image analysis $[3,9,18]$. To get a parameter for the involvement of eosinophils in inflammation that gives more evidence than cell counting, we intended to develop a method for the cell-independent quantification and localization of EGPs in skin biopsies by image analysis of immunostaining.

\section{Material and methods}

\subsection{Material}

Skin punch-biopsies (3-4mm $\varnothing$ ) were taken with informed consent of patients suffering from atopic dermatitis $(n=25)$ or psoriasis $(n=26)$ during of a clinicopathological study on involvement of eosinophils in the inflammatory skin reaction (detailed data in preparation). After immediate fixation in $4 \%$ neutral buffered formalin the material was processed to routine paraffin embedding using automated devices (Pathcentre and Histocentre 640CX51 Shandon, Astmoor, UK). Serial sections with a thickness of $3 \mu \mathrm{m}$ were further processed for immunostaining and image analysis. Biopsies of inflammatory skin diseases with many eosinophils visible in most high power fields $(40 \times \mathrm{ob}-$ jective) of hematoxylin and eosin sections were used as positive controls (e.g., arthropod reactions, bullous pemphigoid).

\subsection{Immunohistochemistry}

Highly sensitive immunohistochemistry was performed with an automated immunostainer (Horizon, DAKO A/S, Glostrup, Denmark) using an indirect streptavidin-biotin method following the standardized MSAPE-protocol of the provider (DAKO).

Four different primary antibodies against eosinophil granule proteins were applied: EG1 against eosinophilic cationic protein $(1: 300$, Kabi Pharmacia, Piscataway, $\mathrm{NJ}$ ); EG2 against both, eosinophilic cationic protein (ECP) and eosinophil-derived neurotoxin / eosinophil protein X (1:300, Kabi Pharmacia); MBP against major basic protein (clone BMK13, 1:10, Cymbus, Southampton, UK) and EPO against eosinophil peroxidase ( 1 : 75, Calbiochem Novabiochem, San Diego, CA). 2 sections were stained with each antibody in each of the 51 cases.
Pretreatment for antigen retrieval was performed by gentle cooking overnight at a temperature of $68^{\circ} \mathrm{C}$ in antigen retrieval solution (DAKO) and in addition with proteinase $\mathrm{K}$; for the EPO-reaction only proteinase $\mathrm{K}$ was used. The positive controls were processed in exactly the same way, negative controls were provided by omission of the primary antibody. Since brown endogeneous pigments (melanin and sometimes siderin) are found in every skin biopsy, brown diamino benzidine (DAB), the usually applied chromogen in image analysis, could not be used. Instead, staining with the bright red newfuchsin was done. To facilitate image analysis, no counterstaining was applied.

\subsection{Image analysis}

Image analysis of the immunostained sections was performed with the software analySIS 3.0 pro, dual monitor version (Soft imaging system, Münster, Germany) and the following hardware components: $\mathrm{BH} 2$ microscope with SplanApo objectives $(20 \times / 0.7$ or $40 \times / 0.7)$ and $1.67 \times$ photoprojective in a $0.3 \times$ tube (Olympus Optical Europe, Hamburg, Germany); high resolution 3CCD colour video camera (DXC 950, Sony Electronics, Japan); Meteor framegrabber (Matrox Electronic Systems, Quebec, Canada) and a Pentium-PC with Windows NT 4.0 workstation.

The immunopositive area fraction (IAF[\%]) was selected as main parameter for the assessment of EGPdeposition. IAF was determined in two different ways: one routine was performed for the evaluation in the total area (MeanIAF of all measured fields) and one for the high power field with the focal maximum area fraction (MaxIAF). IAF was found to be considerably influenced by sampling within the section in preceding measurements. Thus, the following standardized sampling strategies were applied. Concerning MeanIAF, the device of serial field selection was chosen [22]. After determination of the middle of the section at the surface (stratum corneum) by image analytic measurement, sequential images were grabbed using the $20 \times$ objective moving along a central line from the stratum corneum towards subcutis. Two fields on both sides of the central line with a width of $632.55 \mu \mathrm{m}$ and a depth of $464.24 \mu \mathrm{m}$ were measured in up to 10 levels of depth (Fig. 1). The MeanIAF was calculated from the measurements in all non-overlapping fields. For the determination of MaxIAF, a selective sampling strategy was used by scanning the whole section with the $40 \times$ objective. Segmentation was as often performed as nec- 


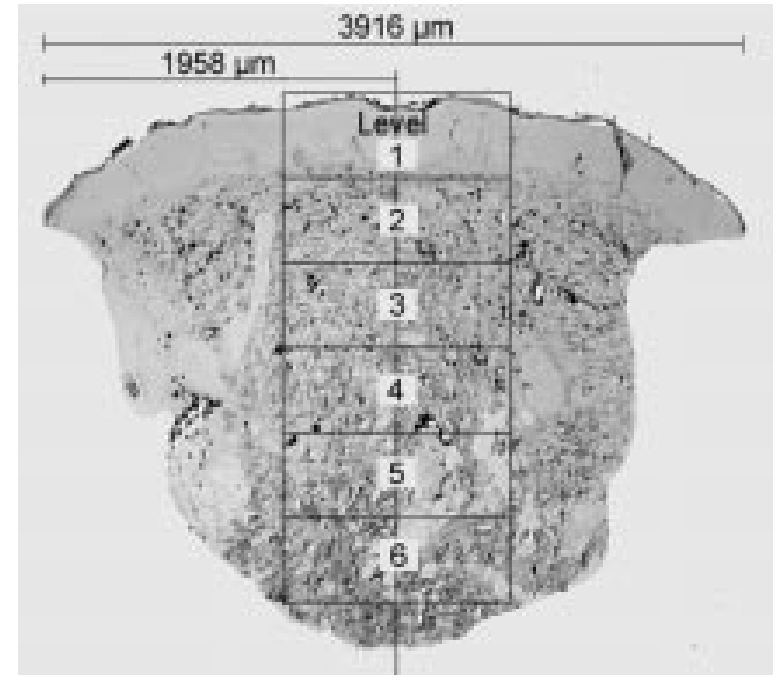

Fig. 1. Sampling strategy. Determination of the mean immunopositive area fraction (MeanIAF) by serial field selection along a central line from stratum corneum to subcutis. Two fields on both sides of the central line with a width of $632.6 \mu \mathrm{m}$ and a depth of $464.2 \mu \mathrm{m}$ were measured in each level of depth.

essary to find the field with the maximum immunoreactivity.

The following image analysis procedure based on methods developed by Smolle [23] and Ruifrok [19] was applied in every field:

1. Additive shading correction.

2. Dual translation of the true colour image to 8-bit grey scale: one in the inverted red channel of the red-green-blue colour space (Rinv) and the other in the saturation channel of the hue-saturationintensity colour space $(\mathrm{S})$.

3. Linear combination of Rinv and $\mathrm{S}$ according to:

$$
\text { GVres }=(\text { GVrinv }+ \text { GVs }) \times 0.5
$$

with GVrinv and GVs representing the grey values in the two translated grey scale pictures, respectively, and GVres representing the resulting grey value of a given pixel.

4. Segmentation of the immunopositive particles in the resulting grey scale picture (Res) by automated thresholding; a module for automated thresholding is implemented in the software, uses $t$-test statistics and is based on the method of Otsu [17].

5. Automatic calculation of the immunopositive area fraction (MeanIAF or MaxIAF) by the software.
6. Automatic calculation of a set of grey value parameters with arbitrary units (0-255) for characterization of the resulting grey scale picture.

For the determination of Mean- and of MaxIAF respectively, we developed two sets of macros to standardize the whole measurement process including: calibration of microscope and camera; presets of image and data handling; determination of the middle of the biopsy; image analysis in every field following steps 1 to 6 as listed above; generation of a table of results and storage of this table together with the original true colour images of all measured fields in a data bank.

Mainly for quality control purposes, the following two grey scale parameters, that were calculated automatically by the software, were further evaluated in addition: total mean grey value (MGROI) and mean grey value of the immunopositive particles that were detected by segmentation (MGIP). For the evaluation of the total area sampling, the following values were calculated out of the field measurements: mean immunopositive area fraction (MeanIAF), total mean grey value (MGROI) and mean grey value of the immunopositive particles (MGIP). Similarly, the following parameters were measured for the assessment of the focal maximum immunoreactivity in high power field (HPF): Immunopositive area fraction (MaxIAF), total mean grey value (MaxMGROI) and mean grey value of the immunopositive particles (MaxMGIP).

\subsection{Evaluation of the method}

Specificity and accuracy of the segmentation in the resulting grey scale picture (Res) of every field were controlled by visual comparison with the immunostaining in the original true colour image. This procedure was included in the macro routines and was facilitated by the dual monitor system, that gives the opportunity to inspect both pictures simultaneously (Fig. 2). Including 51 cases and positive controls in addition, image analysis was performed and evaluated for specificity and accuracy on 6576 fields with the $20 \times$ objective and on 2340 high power fields in 520 sections altogether.

Reproducibility of the results was evaluated by the following experiments using sections of the positive controls:

(1) Repeated measurements of the same immunostained section on different days (REPIMAGE). To look for the variability of sampling within the section 

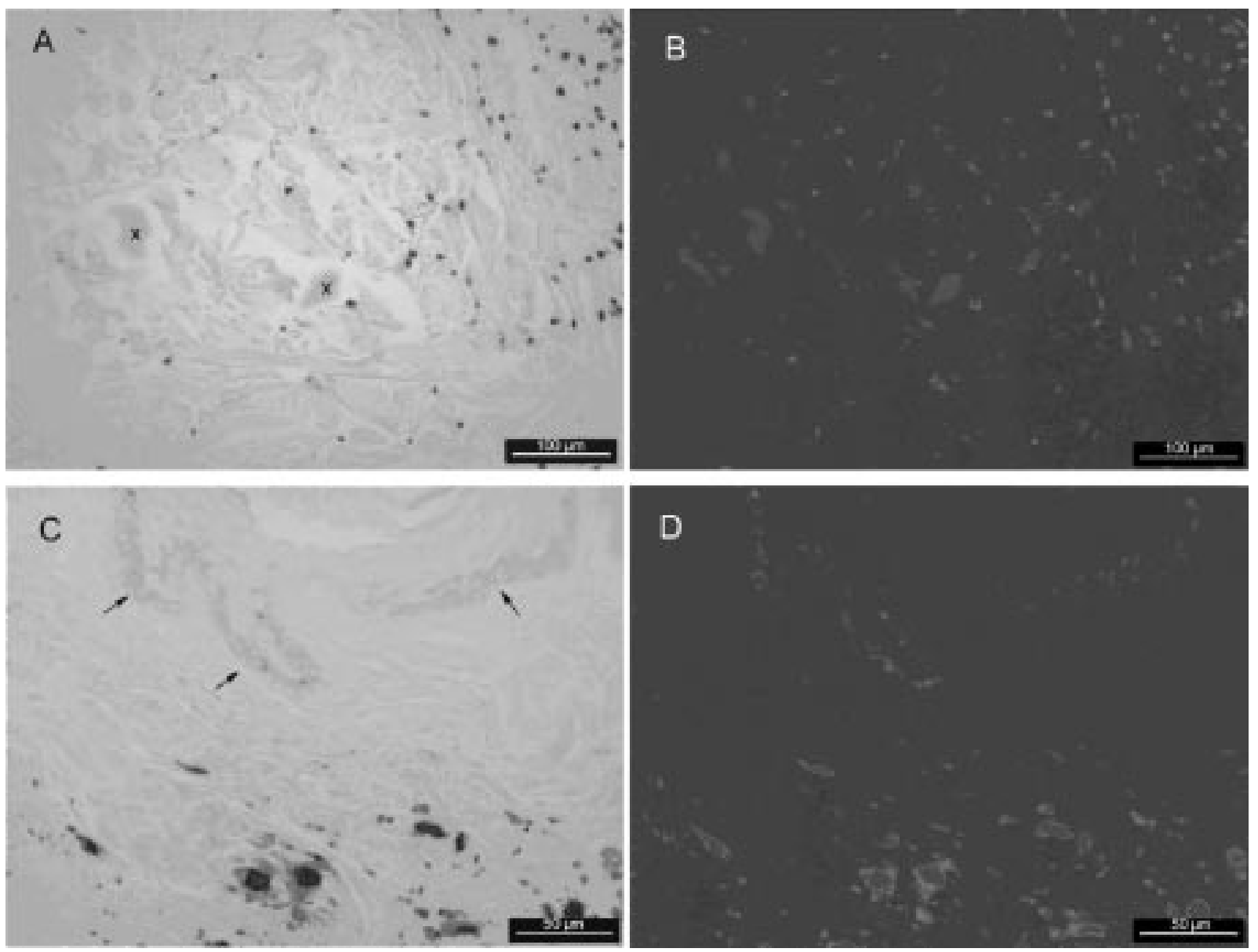

Fig. 2. Specificity and accuracy of the segmentation. Two examples with original true colour pictures showing red immunostained particles $(\mathrm{A}$ and $\mathrm{C}$ ) and the result of cell-independent segmentation in the grey scale picture after colour translation, linear combination and automated thresholding marked by red overlay colour (B and D). Note that unwanted detection of faint background staining ( $\mathrm{x}$ in $\mathrm{A})$ and brown endogeneous melanin pigment ( $\uparrow$ in $\mathrm{C}$ ) could be avoided.

and of image analysis, 6 immunostained sections $(2 \times$ $\mathrm{EG} 1,2 \times \mathrm{EG} 2,1 \times \mathrm{MBP}$ and $1 \times \mathrm{EPO})$ were repeatedly measured. Each section was analyzed 10 times with the routine for the assessment of the total area (parameter MGROI, MGIP and MeanIAF) and 10 times with the routine for the evaluation of focal maximum immunoreactivity (parameter MaxMGROI, MaxMGIP and MaxIAF, see Section 2.3).

(2) Interobserver variability. Pairwise independent measurements of the same immunostained section were performed by two of the authors (K.F. and P.K.) with the routine for the assessment of the total area (parameter MGROI, MGIP and MeanIAF).

(3) Measurements of sequential sections of the same biopsy stained with the same antibody on different occasions (VARTOTAL). For each antibody, one biopsy was selected, and sequential sections were cut. Im- munostaining followed by image analysis of 3 sections (one for each antibody) was performed about once per week within a period of 16 weeks in total to look for the total variation of the mean grey values MGROI and MGIP and the mean immunopositive area fraction IAF during a longer period of time. As multiple sections out of one biopsy were evaluated, the results of this experiment are influenced by sampling within the biopsy in addition to sampling within the section (compare REPIMAGE-experiment). Immunostaining and image analysis contribute to the variability of the results, but each showed different effects on the measured parameters in pre-experiments. Mean total grey value (MGROI) is likely to be least of all influenced by sampling, and intensity of immunostaining has minor effects, if the immunostained area fraction is small. Thus, variation of MGROI can be espe- 
cially considered as a parameter for the overall performance and stability of the image analytic system and of background staining. In contrast, mean grey value of the immunopositive particles (MGIP) may depend to some degree on sampling, but is mostly influenced by the intensity of immunostaining and, in addition, e.g., by threshold setting. Mean immunopositive area fraction (MeanIAF) was found to be most of all influenced by sampling due to inhomogeneous distribution of the immunostained particles within tissue.

\section{Results}

\subsection{Specificity, accuracy and ergonomics}

As shown in Fig. 2, a very accurate correspondence between the immunostained particles in the true colour pictures (Fig. 2(A, C)) and the particles detected by image analysis (red overlay in Fig. 2(B, D) was achieved. In 520 sections of inflammatory skin diseases that were evaluated so far, the automatic segmentation was found to be specific by visual comparison between immunostaining and the result of segmentation in 8916 fields (6576 fields with the $20 \times$ objective and 2340 high power fields). Especially unwanted detection of unspecific, but to some degree unavoidable background staining was not seen (Fig. 2(A, B)). In addition, undesirable segmentation of brown endogeneous pigment (mostly melanin, sometimes siderin) could be nearly completely avoided (Fig. 2(C, D)). Only a few heavily pigmented melanin or dust particles sometimes had to be interactively. This correction step was routinely included in the two sequences of macros and was the only interactive one in the image analytic procedure. Concerning ergonomics, the whole image analytic process for one field than 10 seconds on our system, including grabbing, shading correction, image translations/-combination, automated thresholding, particle detection and generation of the table of results. More time consuming was the standardized sampling procedure within the section including orientation of the slice, determination of the starting point and changing of fields, as well as elimination of artefacts if necessary and storage in the data bank. However, a measurement of a whole section with about 14 fields can be done within 7 minutes by a trained person.

\subsection{Reproducibility}

\subsubsection{Repeated measurements of the same immunostained section on different days (REPIMAGE)}

120 measurements in total were performed on $6 \mathrm{im}$ munostained sections. The results are summarized in Table 1 . Very little variability with CV values far below $1 \%$ was found in these repeated measurements for the mean grey values of the total area (MGROI) and of the field with the maximum immunoreactivity (MaxMGROI). As these parameters are least influenced by sampling, this speaks in favour of a good overall performance and stability of the image analytic system. Moreover, the variability of the mean grey values of the immunopositive particles (MGIP; MaxMGIP) and of the area fraction parameters (MeanIAF; MaxIAF) was very acceptable as well $(\mathrm{CV} \leqslant 5 \%)$.

\subsubsection{Interobserver variability}

On 12 sections, three for each of the four antibodies (EG1, EG2, MBP and EPO), pairwise measurements in 72 fields in total were independently performed by two observers. The results concerning the mean grey value of the total area (MGROI) and of the immunopositive particles (MGIP) as well as the mean immunopositive area fraction (MeanIAF) are shown in Table 2. It can be seen that the deviation of the two measurements from the mean is small and below $5 \%$ for all measurements and parameters. As in the REPIMAGE experiment, the smallest variation was found for MGROI, this parameter appears to be nearly free of observer bias. Regarding MGIP and MeanIAF, the differences between the observers are slightly greater, but the concordance is still very satisfactory.

\subsubsection{Measurements of sequential sections of the same biopsy on different occasions (VARTOTAL)}

44 sequential sections out of 3 different biopsies were immunostained with the antibodies EG1 $(n=$ $15)$, EG2 $(n=15)$ or MBP $(n=14)$ and evaluated within a 16-weeks period by image analysis (554 fields). As can be seen in Fig. 3, the variability of the mean grey values of the total area (MGROI) was very low for immunostaining with each of the three antibodies and not exceeding $\mathrm{CV}$ values of $2.1 \%$. More, but overall very acceptable variability regarding a 16 weeks period was found for the mean grey values of the immunopositive particles (MGIP) with CVs of about $6 \%$ (Fig. 3). Most variation was found for the immunopositive area fraction (IAF) in this as- 
Table 1

REPIMAGE: Repeated measurements of the same immunostained section of different days

\begin{tabular}{|c|c|c|c|c|c|c|}
\hline \multirow[t]{2}{*}{ Antibody } & \multicolumn{3}{|c|}{$\begin{array}{l}\text { Total area } \\
(n=60)\end{array}$} & \multicolumn{3}{|c|}{$\begin{array}{c}\text { High power field } \\
\text { with maximum immunoreactivity } \\
(n=60)\end{array}$} \\
\hline & $\begin{array}{c}\text { MGROI } \\
\text { Mean } \pm \mathrm{SD} \\
\mathrm{CV} \\
\end{array}$ & $\begin{array}{c}\text { MGIP } \\
\text { Mean } \pm \text { SD } \\
\text { CV } \\
\end{array}$ & $\begin{array}{c}\text { MeanIAF } \\
\text { Mean } \pm \text { SD } \\
\text { CV } \\
\end{array}$ & $\begin{array}{c}\text { MaxMGROI } \\
\text { Mean } \pm \text { SD } \\
\mathrm{CV} \\
\end{array}$ & $\begin{array}{c}\text { MaxMGIP } \\
\text { Mean } \pm \text { SD } \\
\text { CV } \\
\end{array}$ & $\begin{array}{c}\text { MaxIAF } \\
\text { Mean } \pm \text { SD } \\
\text { CV } \\
\end{array}$ \\
\hline EG1-I & $\begin{array}{c}68.04 \pm 0.27 \\
0.4 \%\end{array}$ & $\begin{array}{c}160.83 \pm 5.84 \\
3.6 \%\end{array}$ & $\begin{array}{c}0.52 \pm 0.01 \\
2.0 \%\end{array}$ & $\begin{array}{c}72.21 \pm 0.29 \\
0.4 \%\end{array}$ & $\begin{array}{c}127.57 \pm 1.87 \\
1.5 \%\end{array}$ & $\begin{array}{c}2.63 \pm 0.08 \\
3.1 \%\end{array}$ \\
\hline EG1-II & $\begin{array}{c}71.98 \pm 0.13 \\
0.2 \%\end{array}$ & $\begin{array}{c}154.45 \pm 1.67 \\
1.1 \%\end{array}$ & $\begin{array}{c}1.54 \pm 0.02 \\
1.5 \%\end{array}$ & $\begin{array}{c}75.29 \pm 0.31 \\
0.4 \%\end{array}$ & $\begin{array}{c}118.99 \pm 1.46 \\
1.2 \%\end{array}$ & $\begin{array}{c}4.25 \pm 0.16 \\
3.7 \%\end{array}$ \\
\hline EG2-I & $\begin{array}{c}69.63 \pm 0.12 \\
0.2 \%\end{array}$ & $\begin{array}{c}175.19 \pm 2.58 \\
1.5 \%\end{array}$ & $\begin{array}{c}1.13 \pm 0.01 \\
0.9 \%\end{array}$ & $\begin{array}{c}74.93 \pm 0.56 \\
0.8 \%\end{array}$ & $\begin{array}{c}123.20 \pm 2.56 \\
2.1 \%\end{array}$ & $\begin{array}{c}4.91 \pm 0.19 \\
3.9 \%\end{array}$ \\
\hline EG2-II & $\begin{array}{c}71.78 \pm 0.44 \\
0.6 \%\end{array}$ & $\begin{array}{c}171.29 \pm 5.76 \\
3.4 \%\end{array}$ & $\begin{array}{c}1.81 \pm 0.07 \\
3.9 \%\end{array}$ & $\begin{array}{c}75.52 \pm 0.42 \\
0.6 \%\end{array}$ & $\begin{array}{c}120.18 \pm 1.58 \\
1.3 \%\end{array}$ & $\begin{array}{c}5.34 \pm 0.22 \\
4.1 \%\end{array}$ \\
\hline MBP & $\begin{array}{c}70.11 \pm 0.34 \\
0.5 \%\end{array}$ & $\begin{array}{c}144.78 \pm 4.45 \\
3.1 \%\end{array}$ & $\begin{array}{c}0.69 \pm 0.03 \\
5.0 \%\end{array}$ & $\begin{array}{c}74.40 \pm 0.31 \\
0.4 \%\end{array}$ & $\begin{array}{c}113.62 \pm 1.39 \\
1.2 \%\end{array}$ & $\begin{array}{c}3.05 \pm 0.13 \\
4.2 \%\end{array}$ \\
\hline EPO & $\begin{array}{c}67.38 \pm 0.07 \\
0.1 \%\end{array}$ & $\begin{array}{c}149.32 \pm 1.64 \\
1.1 \%\end{array}$ & $\begin{array}{c}0.11 \pm 0.005 \\
4.5 \%\end{array}$ & $\begin{array}{c}68.37 \pm 0.12 \\
0.2 \%\end{array}$ & $\begin{array}{c}131.10 \pm 1.80 \\
1.4 \%\end{array}$ & $\begin{array}{c}0.69 \pm 0.01 \\
2.0 \%\end{array}$ \\
\hline
\end{tabular}

MGROI: mean grey value of the field(s) [arbitrary units, $0-255$ ].

MGIP: mean grey value of all immunopositive particles [arbitrary units, 0-255].

IAF: immunopositive area fraction in the field(s) [\%]. $\mathrm{CV}=$ coefficient of variation $=\mathrm{SD} / \mathrm{Mean} * 100[\%]$.

Table 2

Interobserver variability

\begin{tabular}{|c|c|c|c|c|c|c|c|c|c|}
\hline \multirow[t]{2}{*}{ Antibody } & \multicolumn{3}{|c|}{ MGROI } & \multicolumn{3}{|c|}{ MGIP } & \multicolumn{3}{|c|}{ MeanIAF } \\
\hline & Observer 1 & Observer 2 & $\begin{array}{l}\text { Deviation } \\
\text { from mean }\end{array}$ & Observer 1 & Observer 2 & $\begin{array}{l}\text { Deviation } \\
\text { from mean }\end{array}$ & Observer 1 & Observer 2 & $\begin{array}{l}\text { Deviation } \\
\text { from mean }\end{array}$ \\
\hline EG1-I & 74.91 & 75.09 & $0.1 \%$ & 115.82 & 116.89 & $0.5 \%$ & 3.51 & 3.67 & $2.2 \%$ \\
\hline EG1-II & 73.27 & 73.09 & $0.1 \%$ & 121.36 & 122.86 & $0.6 \%$ & 3.63 & 3.53 & $1.4 \%$ \\
\hline EG1-III & 73.69 & 73.31 & $0.3 \%$ & 120.66 & 121.78 & $0.5 \%$ & 3.77 & 3.64 & $1.8 \%$ \\
\hline EG2-I & 65.83 & 65.52 & $0.2 \%$ & 164.91 & 155.49 & $2.9 \%$ & 0.31 & 0.29 & $3.8 \%$ \\
\hline EG2-II & 65.62 & 65.46 & $0.1 \%$ & 176.23 & 166.81 & $2.7 \%$ & 0.43 & 0.42 & $1.5 \%$ \\
\hline EG2-III & 65.55 & 65.39 & $0.1 \%$ & 170.76 & 162.36 & $2.5 \%$ & 0.35 & 0.34 & $1.7 \%$ \\
\hline MBP-I & 68.13 & 67.94 & $0.1 \%$ & 129.17 & 124.37 & $1.9 \%$ & 0.21 & 0.22 & $1.8 \%$ \\
\hline MBP-II & 67.41 & 67.16 & $0.2 \%$ & 134.77 & 130.06 & $1.8 \%$ & 0.34 & 0.35 & $1.3 \%$ \\
\hline MBP-III & 65.33 & 65.21 & $0.1 \%$ & 147.90 & 140.41 & $2.6 \%$ & 0.20 & 0.21 & $2.5 \%$ \\
\hline EPO-I & 65.76 & 65.48 & $0.2 \%$ & 138.11 & 134.32 & $1.4 \%$ & 0.12 & 0.11 & $4.3 \%$ \\
\hline EPO-II & 66.54 & 66.18 & $0.3 \%$ & 155.77 & 144.20 & $3.9 \%$ & 0.25 & 0.24 & $2.2 \%$ \\
\hline EPO-III & 67.40 & 67.02 & $0.3 \%$ & 138.40 & 135.09 & $1.2 \%$ & 0.35 & 0.34 & $2.0 \%$ \\
\hline
\end{tabular}

MGROI: mean grey value of the field(s) [arbitrary units, $0-255$ ].

MeanIAF: mean immunopositive area fraction in the field(s) [\%].

MGIP: mean grey value of all immunopositive particles [arbitrary units, 0-255].

say of multiple sequential sections (Fig. 4). For a biopsy with rather large content of eosinophilic granule proteins (biopsy immunostained with EG1), this was less remarkable with a $\mathrm{CV}$ of about $8 \%$, and by calculation of a running mean (RM) very accept- able variation could be achieved (mean $\mathrm{CV}$ of $\mathrm{RM}$ in all measurements $2.7 \%$, see Fig. 4 ; maximum CV of $\mathrm{RM}$ in any of the measurements $2.8 \%$, data not shown). However, in the two biopsies with rather low total content of eosinophilic granule protein, immunos- 


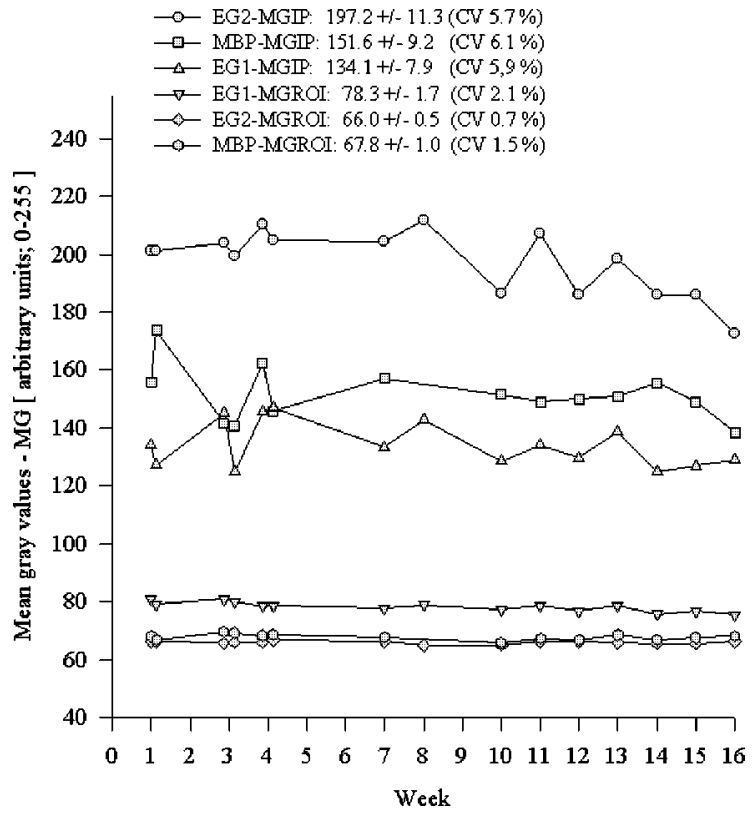

Fig. 3. VARTOTAL-MG: Mean grey values (MG) in 44 sequential sections immunostained with the antibodies EG1 $(n=15)$, EG2 ( $n=15$ ) or MBP $(n=14)$ within a 16-week period. (MGIP: MG of the immunopositive particles; MGROI: MG of the total area. Values in the legend: Mean of all measurements $\pm \mathrm{SD}, \mathrm{CV}=$ coefficient of variation).

tained with EG2 and MBP, more relative variation of IAF was seen. (CV-EG2: $39.4 \%$; CV-MBP: $32 \%$. Mean/maximum CV of RM for EG2: $9.1 \% / 10.7 \%$ and for MBP: $14.8 \% / 14.2 \%$, respectively; see Fig. 4.) This is not astonishing if the very low absolute values are taken into account (MeanIAF-EG2 $0.33 \%$, MeanIAFMBP $0.34 \%$ ). Moreover, as could be assessed by additional visual comparison of the sequential sections, this variation was mainly due to inhomogeneous distribution of the overall small amount of granule proteins between the sections.

\section{Discussion}

Extracellular tissue deposition of reactive oxygen species and eosinophilic granule proteins (EGPs) is the most important local effector function of eosinophilic granulocytes $[7,13,24]$. However, a quantitative morphometric approach for the cell-independent assessment of EGPs in situ is lacking to date. Even recent assays using image analysis for cell counting of eosinophils $[3,9,18]$ do no consider that eosinophils are no longer identifiable in tissue after complete activation and degranulation, with the consequence

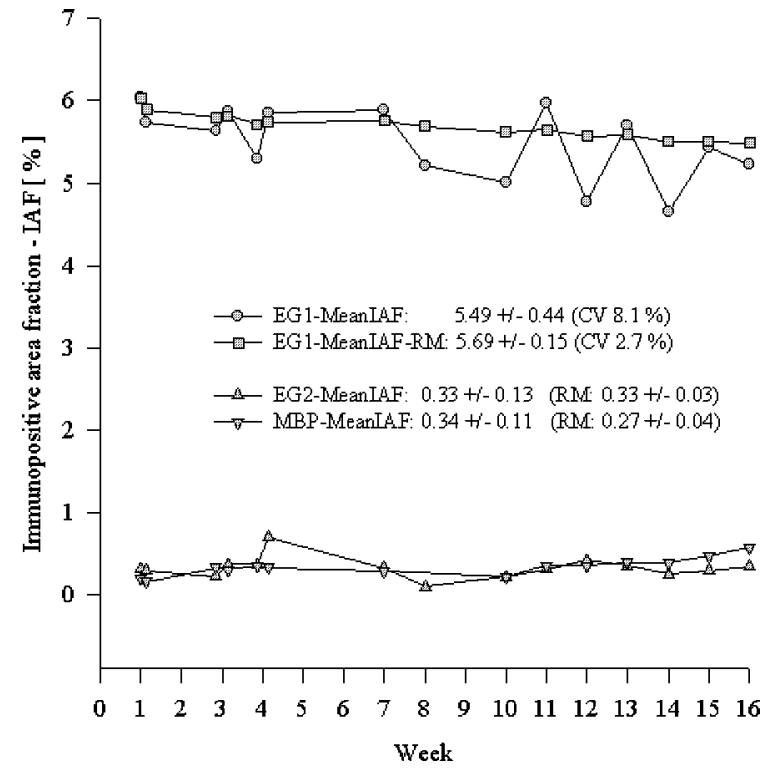

Fig. 4. VARTOTAL-IAF: Mean immunopositive area fraction (MeanIAF) in 44 sequential sections immunostained with the antibodies EG1 $(n=15)$, EG2 $(n=15)$ or MBP $(n=14)$ within a 16-weeks period. (Values in the legend: Mean of all measurements $\pm \mathrm{SD} ; \mathrm{CV}=$ coefficient of variation; $\mathrm{RM}=$ running mean.)

that eosinophil involvement in inflammation cannot be judged by the number of intact eosinophils. This has been clearly shown by immunofluorescence studies, especially of inflammatory skin diseases [14,15]. However, these immunofluorescence studies are semiquantitative at best, scoring visual analogue grades, that are a complex composition of intensity and distribution of immunofluorescence [6]. Ordinal scales of that kind are likely to be less reproducible and objective than quantitative morphometry [2,25] and probably as a consequence, results of such immunofluorescence studies have not been published by many different groups.

Quantitative immunohistochemistry using image analysis is increasingly applied in pathology, e.g., for the assessment of tumour cell proliferation or the estimation of hormone receptor expression in oncology [4]. Most publications concerning the quantitative evaluation of immunostaining have found good correlation with biochemical results, where careful standardization of the assays was performed, e.g., in molecular markers of breast cancer [5]. Moreover, in contrast to biochemical analyses or immunoassays for the determination of ECP [26], quantitative immunohistochemistry allows studying the local organization of inflammation and their regulation, even in small samples of tissue. This is a prerequisite for pat- 
tern analysis, which represents the basis for dermatohistopathological analysis of inflammatory skin diseases [1]. Thus, we intended to develop a method for the cell-independent quantification and localization of eosinophilic granule proteins based on automated image analysis of highly sensitive immunostaining, that gives more evidence for the involvement of eosinophils in inflammation than cell counting, is more reproducible and objective than immunofluorescence and allows one, in contrast, e.g., to biochemical assays and flow cytometry to analyse the local organization and regulation of inflammation in skin and other tissues.

Many different methods have been applied for the immunohistochemical quantification of cellular properties based on nuclear, cytoplasmatic or membranous staining, mainly intending to quantify the immunolabelled area fraction and/or the staining intensity by densitometry [11]. However, applications for the cell-independent evaluation of immunostaining, possibly suitable for the quantification of eosinophilic granule proteins are rare. Most interesting was the method successfully used by Furness et al. [10] for the semi-automatic quantification of macrophages in renal biopsies. Similar to this strategy, we selected the immunopositive area fraction (IAF) as main parameter for the assessment of EGP-deposition. The mean as well as the focal maximum IAF were assessed in two different routines. Concerning MeanIAF, the spatial relationships of immunostaining are also of interest. Consequently, we chose the device of serial field selection (see Fig. 1), which gives a cross-sectional representation of the immunostaining distribution within the section for sampling [22]. The main problem concerning the specificity of segmentation was unwanted detection of brown endogeneous pigment in skin (mostly melanin, sometimes siderin). Thus, we found the use of bright red newfuchsin instead of diamino benzidine (DAB), which is the usually applied chromogen in image analysis, to be a prerequisite for successful discrimination between endogeneous pigment and specific immunostaining. Counterstaining, an issue not fully resolved in image analysis [11], is not necessary for this cell-independent assay and could be omitted. Using the analySIS 3.0 pro software, various attempts were made to perform segmentation by interactive thresholding in true colour pictures of the redgreen-blue (RGB), as well as in the hue-saturationintensity (HSI) colour space. However, though it was perfectly possible to perform specific segmentation of the bright red immunostaining avoiding unwanted detection of brown endogeneous pigments, the repro- ducibility was not acceptable. This was mainly due to bias of interactive thresholding, a well-known problem in image analysis $[12,16,20]$. Especially the occurrence of poorly outlined particles and fading of colour at the edges of particles resulted in considerable differences in interactive threshold setting. The use of fixed external thresholds may be a possible solution for this problem [27], but automatic thresholding is the best way to avoid observer bias [21]. Automatic thresholding, based on the method of Otsu [17], is implemented in the analySIS 3.0 pro software and is also available in the widely used public domain image processing program NIH-Image for the Macintosh workstation. However, it is only suitable for grey scale pictures. Consequently, we adapted the methods of Ruifrok [19] and Smolle [23] for our setting by translation of the true colour pictures to grey scale in two different channels, followed by linear combination and finally automated thresholding in the resulting grey scale picture. Out of different approaches, the combination of the inverted red channel of RGB and the saturation channels of HSI was found to be best and highly specific in automatically discriminating between red immunostaining on the one hand and unwanted background as well as brown endogeneous pigment on the other hand (Fig. 2). By visual comparison between immunostaining and the result of segmentation, the automatic segmentation was found to be specific in nearly 9000 fields with low and high content of EGPs that were have measured so far.

The reproducibility of the method was evaluated in three different experiments. The first was done to look mostly for variation caused by sampling within the section and image analysis (REPIMAGE). In addition, we examined interobserver variation in a series of pairwise measurements, and the most comprehensive experiment (VARTOTAL) was performed to look for stability of the measurements within 16 weeks. Significant variations of immunostaining and image analysis are most likely to appear in practice during this rather long of time period. However, as this latter experiment was performed on sequential sections, the variations in these results may not only be caused by the measurement method, but in addition by a possible inhomogeneous distribution of granule protein deposition within the biopsy. Immunopositive area fraction was used as main target parameter for the evaluation of EGPdeposition. Measurements of optical density were not performed for this purpose. Densitometric evaluation of immunohistochemical staining on slides is possible, but there are many problems concerning standardiza- 
tion, e.g., the requirement of corrective factors for section thickness to assure comparability [16]. Instead, we used two different, simple grey value parameters, the mean grey value of the total field area (MGROI) and the mean grey value of the immunopositive particles (MGIP) for quality control purposes. In all three experiments very low variation was found for MGROI (Tables 1, 2, Fig. 3). Concerning MGIP, the variation was similarly acceptable in repeated measurements of the same immunostained section $(\mathrm{CV}<5 \%)$ and concerning interobserver variability (Tables 1 and 2). This speaks in favour of a good overall performance and stability of the automatic image analytic system including thresholding. Similarly, acceptable stability was found for the immunopositive area fraction (IAF) in the REPIMAGE experiment ( $\mathrm{CV} \leqslant 5 \%$; Table 1 ) as well as concerning interobserver variation (Table 2). Differences between the observers regarding MGIP and MeanIAF (Table 2) are most likely due to minor differences in sampling and elimination of dust particles or endogeneous pigment - the only interactive steps in the routine. Taken together, the sampling strategy within the section as well as the total image analytic process appear to be standardized and precise enough to get highly reproducible results throughout repeated measurements.

In the most comprehensive experiment VARTOTAL, the variation of MGROI was found to be very low again (maximum CV 2.1\%, Fig. 3). This speaks in favour of a good standardization of the staining procedure, especially concerning unwanted background staining, as well as a good overall performance and stability of the image analytic system over a longer time period. The mean grey value of the immunostained particles (MGIP) mostly reflects the intensity of immunostaining, even if this parameter is also influenced by image analysis, especially thresholding. Variation of MGIP was acceptable regarding the long observation period of this experiment (CV for MGIP about $6 \%$ over 16 weeks, Fig. 3). In contrast to the mean grey value parameters, the mean immunopositive area fraction (IAF) was found to show more variation in this VARTOTAL-experiment, especially in biopsies with low total amount of granule protein deposition (Fig. 4). As could be assessed by additional visual comparison of the sequential sections, this higher variation was mainly due to inhomogeneous distribution of the small amount of total granule proteins between the sections.

In conclusion, a good accuracy, reproducibility and reliability, even over a long observation period of 16 weeks, could be achieved for the total assay includ- ing immunostaining and image analysis. However, inhomogeneous distribution of eosinophilic granule proteins within tissue has to be taken into account concerning the evaluation of the immunopositive area fraction (IAF), especially in cases with low total amount of protein. As a consequence, sampling within the biopsies and within the sections has to be performed carefully. However, to be able to detect such distributional variation is an advantage of quantitative immunohistochemistry in contrast, e.g., to biochemical assays [5], since it allows evaluating local mechanisms of regulation, e.g., in inflammation, even in small samples of tissue.

Standardized and detailed protocols for sampling, immunostaining and image analysis, as far as possible using automated devices including automated thresholding were found to be crucial. Under these conditions, image analysis of immunostaining using colour translation, linear combination and automated thresholding was found to be suitable for the specific detection and quantification of eosinophilic granule proteins in skin biopsies.

The assay may be suitable for the cell-independent evaluation of immunostaining in other applications as well.

\section{Acknowledgements}

The authors want to thank Kerstin Muth and the laboratory team for excellent technical assistance as well as Dr Hartmut Herrmann, Institute of Biometrics, Hannover Medical University for statistical advice.

\section{References}

[1] A.B. Ackerman, N. Chongchitnant, J. Sanchez, G. Ying, B. Bennin, M. Reichel and M.B. Randall, Histologic Diagnosis of Inflammatory Skin Diseases. An Algorithmic Method Based on Pattern Analysis, 2 edn, Williams \& Wilkins, Baltimore, 1997.

[2] J.P.A. Baak and J.C. Fleege, Anwendungen von quantitativen Methoden in der diagnostischen Pathologie, Verh. Dtsch. Ges. Pathol. 74 (1990).

[3] J.W. Bishop, Quantification of tissue eosinophils and lymphocytes in histologic sections, Mod. Pathol. 11 (1998), 12471251.

[4] G. Brugal, Quantitative microscopy and tumour cell proliferation, Bull. Cancer 82 (1995), 511s-517s.

[5] C. Charpin, P.M. Martin, B. De Victor, M.N. Lavaut, M.C. Habib, L. Andrac and M. Toga, Multiparametric study (SAMBA 200) of estrogen receptor immunocytochemical assay in 400 human breast carcinomas: analysis of estrogen receptor distribution heterogeneity in tissues and correlations with dextran coated charcoal assays and morphological data, Cancer Res. 48 (1988), 1578-1586. 
[6] J.F. Cheng, N.L. Ott, E.A. Peterson, T.J. George, M.J. Hukee, G.J. Gleich and K.M. Leiferman, Dermal eosinophils in atopic dermatitis undergo cytolytic degeneration, J. Allergy Clin. Immunol. 99 (1997), 683-692.

[7] J. Elsner and A. Kapp, Regulation and modulation of eosinophil effector functions, Allergy 54 (1999), 15-26.

[8] W.L. Epstein, Eosinophilia, N. Engl. J. Med. 338 (1998), 15921600.

[9] A. Furgal and J.A. Litwin, Distribution of eosinophils along and across successive segments of the rat digestive tract: a quantitative study, Folia Histochem. Cytobiol. 35 (1997), 155163.

[10] P.N. Furness, L. Rogers-Wheatley and K.P. Harris, Semiautomatic quantitation of macrophages in human renal biopsy specimens in proteinuric states, J. Clin. Pathol. 50 (1997), 118-122.

[11] F. Giroud, C. Perrin, M.P. Montmasson, R. Kiss and I. Camby, Tutorial on quantitative immunocytochemistry and immunohistochemistry. Handout 5th Conference of the European Society for Analytical Cellular Pathology, May 25-29, Oslo, Norway, 1997.

[12] R. Jagoe, J.H. Steel, V. Vucicevic, N. Alexander, S. Van Noorden, R. Wootton and J.M. Polak, Observer variation in quantification of immunocytochemistry by image analysis, Histochem. J. 23 (1991), 541-547.

[13] P. Kiehl and A. Kapp, Gewebeeosinophilie und lokale Deposition von Eosinophilen-spezifischen Granulaproteinen. Regulation und Bedeutung für die Entzündungsantwort bei der atopischen Dermatitis und anderen entzündlichen Dermatosen, Hautarzt 49 (1998), 176-183.

[14] K.M. Leiferman, A current perspective on the role of eosinophils in dermatologic diseases, J. Am. Acad. Dermatol. 24 (1991), 1101-1112.

[15] K.M. Leiferman, S.J. Ackerman, H.A. Sampson, H.S. Haugen, P.Y. Venencie and G.J. Gleich, Dermal deposition of eosinophil-granule major basic protein in atopic dermatitis. Comparison with onchocerciasis, N. Engl. J. Med. 313 (1985), 282-285.
[16] M. Oberholzer, M. Ostreicher, H. Christen and M. Bruhlmann, Methods in quantitative image analysis, Histochem. Cell Biol. 105 (1996), 333-355.

[17] N. Otsu, A threshold selection method from grey level histogram, IEEE Trans. SMC 9 (1979), 62-66.

[18] R. Pawliczak, M.L. Kowalski, M. Danilewicz, M. WagrowskaDanilewicz and A. Lewandowski, Distribution of mast cells and eosinophils in nasal polyps from atopic and nonatopic subjects: a morphometric study, Am. J. Rhinol. 11 (1997), 257262.

[19] A.C. Ruifrok, Quantification of immunohistochemical staining by color translation and automated thresholding, Anal. Quant. Cytol. Histol. 19 (1997), 107-113.

[20] J.C. Russ, The Image Processing Handbook, 2nd edn, CRC Press, Inc, Boca Raton, FL, 1995.

[21] J.C. Russ, Thresholding images, J. Comput. Assist. Microsc. 7 (1995), 141-164.

[22] J.M. Skinner, Y. Zhao, B. Coventry and J. Bradley, Video image analysis in pathology, Dis. Markers 11 (1993), 53-70.

[23] J. Smolle, Optimization of linear image combination for segmentation in red-green-blue images, Anal. Quant. Cytol. Histol. 18 (1996), 323-329.

[24] S. Sur, C.R. Adolphson and G.J. Gleich, Eosinophils. Biochemical and cellular aspects, in: Allergy. Principles and Practice, E. Middleton, Jr, C.E. Reed, E.F. Ellis, N.F. Adkinson, Jr, J.W. Yunginger and W. Busse, eds, Mosby, St. Louis, 1993, pp. 169-200.

[25] L.D. True, Morphometric applications in anatomic pathology, Hum. Pathol. 27 (1996), 450-467.

[26] P. Venge and J. Bystrom, Eosinophil cationic protein (ECP), Int. J. Biochem. Cell Biol. 30 (1998), 433-437.

[27] F. Willemse, M. Nap, S.C. Henzen-Logmans and H.F. Eggink, Quantification of area percentage of immunohistochemical staining by true color image analysis with application of fixed thresholds, Anal. Quant. Cytol. Histol. 16 (1994), 357-364. 


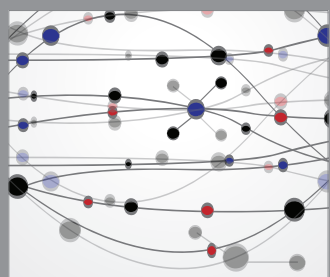

The Scientific World Journal
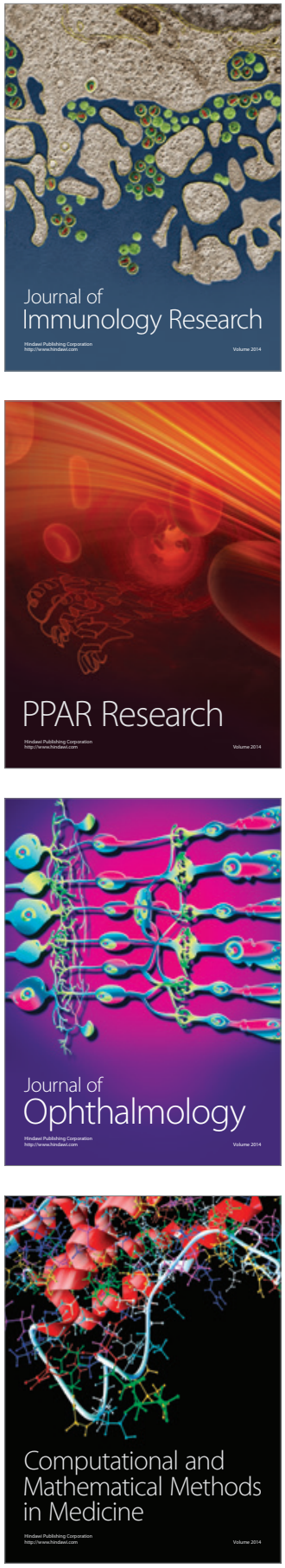

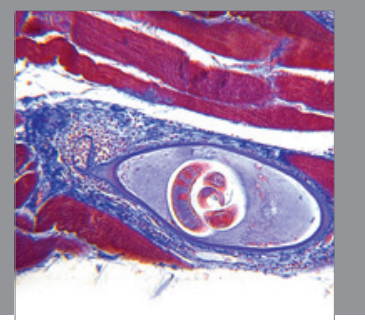

Gastroenterology

Research and Practice
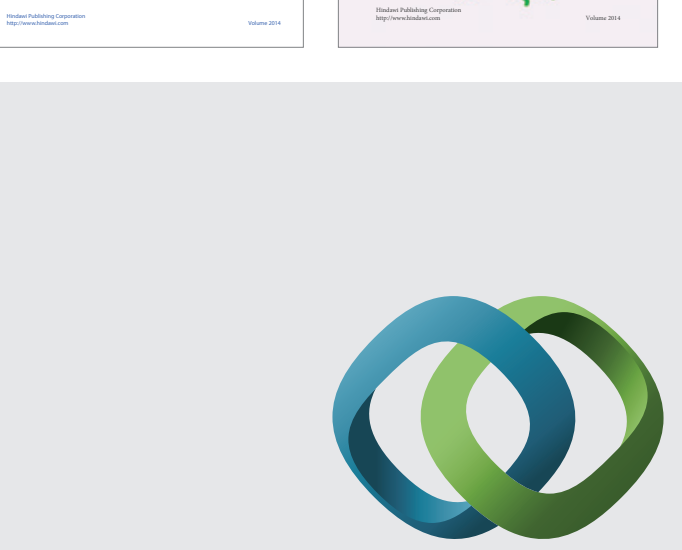

\section{Hindawi}

Submit your manuscripts at

http://www.hindawi.com
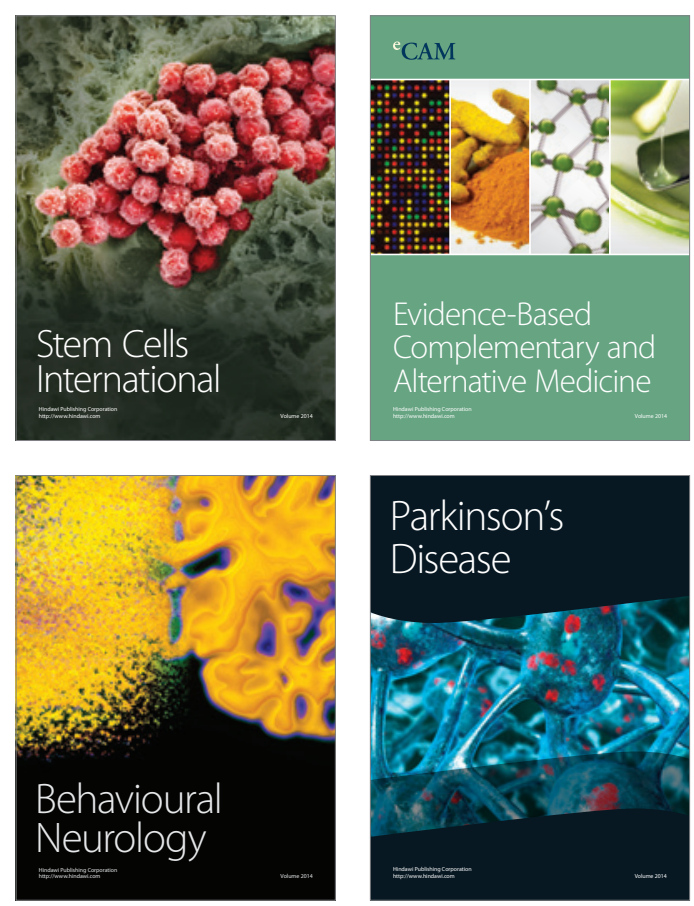

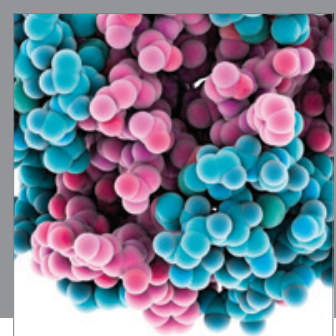

Journal of
Diabetes Research

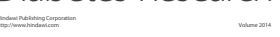

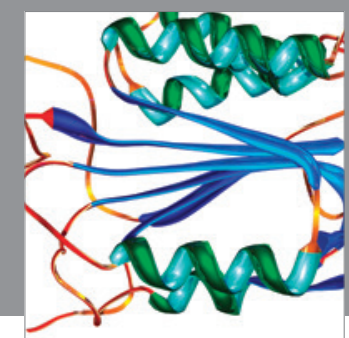

Disease Markers
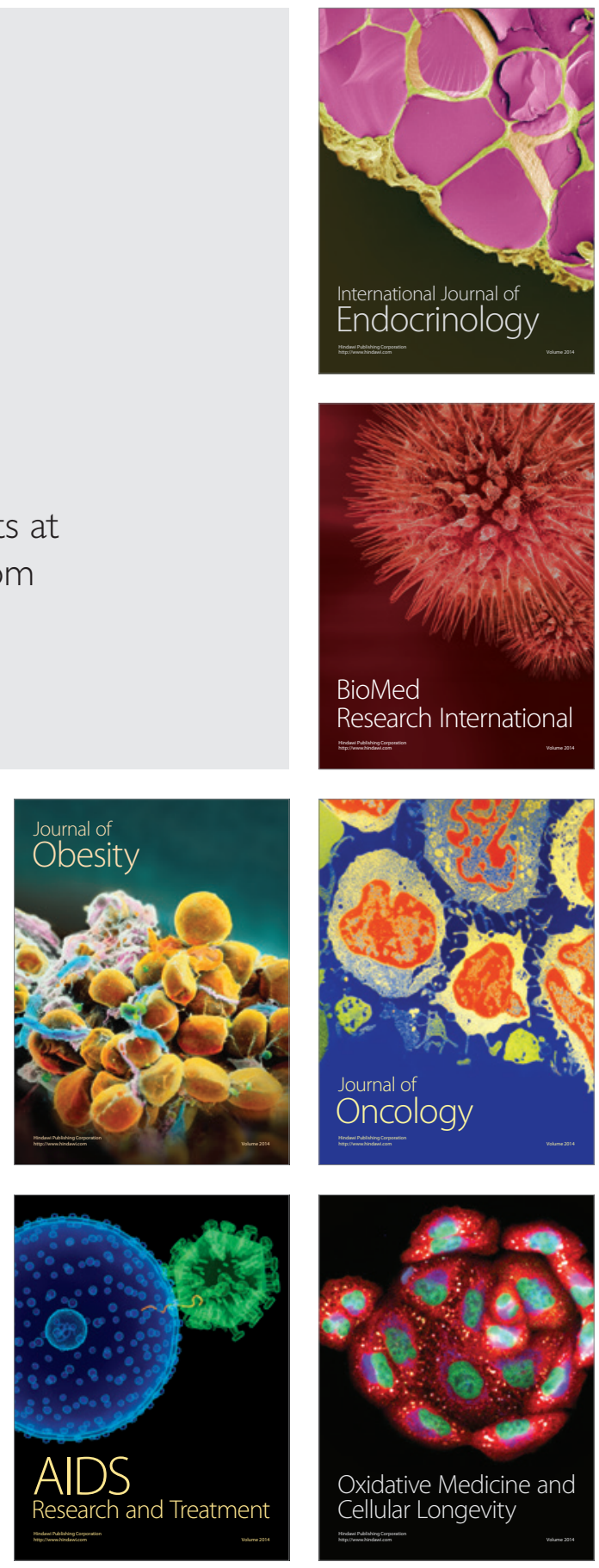